\title{
Manejo quirúrgico de la poliposis nasosinusal
}

\section{Surgical management of sinonasal polyposis}

Rodrigo Arregui $\mathrm{V}^{1}$, Jaime Osorio $\mathrm{M}^{1}$, Andrés Readi $\mathrm{S}^{1}$, Tamara Barría E².

\section{RESUMEN}

Introducción: La poliposis nasosinusal es un proceso inflamatorio crónico de la mucosa rinosinusal, de etiología desconocida, y que requiere de tratamiento quirúrgico en más de $50 \%$ de los pacientes.

Objetivo: Evaluar los resultados de la cirugía endoscópica en pacientes con poliposis nasosinusal en el Hospital Barros Luco-Trudeau.

Material y método: Estudio retrospectivo de 180 fichas clínicas de pacientes operados en este hospital en el período 1999-2008.

Resultados: La distribución según sexo fue de $63 \%$ varones y $37 \%$ mujeres ( $p$ $<0,001)$ y el promedio etario, de 44,9 años. El motivo principal de consulta fue la obstrucción nasal. Un tercio de los pacientes tenía poliposis masiva. El 17\% se asoció con asma. Más del $50 \%$ de los pacientes usó corticoides inhalados en el preoperatorio. En el 58\% se realizó cirugía endoscópica hasta etmoides anterior. No hubo complicaciones mayores en esta serie estudiada. La recidiva global fue de $24 \%$. El promedio de meses libres de recidiva fue 46,7 meses (IC 95\%: 37,5-55,5), con una recidiva de $25 \%$ al año de seguimiento. No se encontró asociación entre recidiva y los factores de riesgo estudiados.

Discusión: En comparación con la literatura, se observa similares características demográficas. Sin embargo, los factores de riesgo de recidiva no fueron significativos, aparentemente debido a una baja prevalencia y a pérdida del seguimiento. La tasa de recidiva aumenta con el tiempo, motivo por el cual se requiere un seguimiento a largo plazo.

Conclusiones: No se detectó asociación entre recidiva y los factores de riesgo clásicamente descritos en la literatura. Las recidivas fueron tardías y se obtuvieron tasas de recidiva similares a lo publicado en la literatura.

Palabras clave: Cirugía endoscópica, poliposis nasosinusal, rinosinusitis crónica.

\section{ABSTRACT}

Introduction: Sinonasal polyposis is a chronic inflammatory process rhinosinusal mucosa of unknown etiology, which in over $50 \%$ of patients requires surgical treatment.

Aim: To evaluate the results of endoscopic surgery in patients with sinonasal polyposis in the Barros Luco-Trudeau Hospital.

\footnotetext{
1 Médico del Servicio de Otorrinolaringología, Hospital Barros Luco-Trudeau, Servicio de Salud Metropolitano Sur.

2 Interna de Medicina, Universidad Andrés Bello.
} 
Material and method: Retrospective study of 180 medical records of patients operated at this hospital from 1999 to 2008.

Results: The sexual distribution was $63 \%$ male and $37 \%$ women $(p<0.001)$. The average age was 44.9 years. The chief complaint was nasal obstruction. A third of the patients had massive polyposis. The $17 \%$ was associated with asthma. Over $50 \%$ of patients used inhaled corticosteroids preoperatively. In 58\% performed endoscopic surgery to anterior ethmoid. There were no major complications in these series studied. The overall recurrence was $24 \%$. The average number of months free of recurrence was 46.7 months (95\% Cl 37.5 to 55.5 ) with a recurrence of $25 \%$ per year of follow up. We found no association between recurrence and the risk factors studied.

Discussion: Comparing with the literature, we see the same demographic characteristics but risk factors for recurrence are not significant, apparently due to the low prevalence and loss of follow-up. The recurrence rate is increased over time and therefore requires a long-term monitoring.

Conclusions: We found no association between recurrence and the risk factors classically described in literature. Recurrences were late and we had similar recurrence rates to publish in the literature.

Key words: Endoscopic surgery, sinonasal polyposis, chronic rhinosinusitis.

\section{INTRODUCCIÓN}

La poliposis nasal es un proceso inflamatorio crónico que afecta a las mucosas de las fosas nasales y los senos paranasales. Su etiología es desconocida, no mediada por inmunoglobulina $E$ ( $\mathrm{IgE}$ ) sistémica ${ }^{1}$. Actualmente se considera que esta enfermedad constituye un subgrupo dentro de las rinosinusitis crónicas ${ }^{2,5}$. Los pólipos están formados por tejido conectivo laxo, edema, células inflamatorias, algunas glándulas y capilares. Los eosinófilos son las células inflamatorias que aparecen con mayor frecuencia en los pólipos nasales. Localmente predomina la interleuquina-5 (IL-5), Io que conlleva que los eosinófilos estén más activos y sobrevivan durante más tiempo. Asociado a esto se ha descrito que la IgE específica contra Staphylococcus o superantígenos actuaría como moduladora de esta enfermedad1-6.

Se estima que la prevalencia en la población general es de $1 \%$ a $4 \%$, dependiendo del método de estudio empleado. Representa el $5 \%$ de las consultas en Otorrinolaringología ${ }^{1,2}$.

Los objetivos del tratamiento son eliminar 0 reducir el tamaño de los pólipos, eliminar los síntomas de rinitis, restablecer la respiración y el olfato, y prevenir la recurrencia 4 .

Dentro de las opciones de tratamiento médico que han sido respaldadas por la evidencia, están los corticoides inhalados y los corticoides sisté$\operatorname{micos}^{2,3}$.

El tratamiento quirúrgico estaría indicado en: a) poliposis masiva; b) falta de respuesta al tratamiento médico; c) sospecha de rinosinusitis fúngica alérgica; y d) frente a recurrencia a pesar de la terapia médica. Más del $50 \%$ de los pacientes con poliposis requerirá de una intervención quirúrgica' ${ }^{14,21}$.

En las últimas dos décadas la cirugía endoscópica (CE) se ha consolidado para el manejo de la poliposis, ya que presenta una baja tasa de complicaciones graves, y exhibe resultados satisfactorios. Sus objetivos son recuperar la mucosa enferma, facilitar la ventilación y el aclaramiento mucociliar, y mejorar la llegada del corticoide tópico a la mucosa nasal ${ }^{1-4,28}$.

En diversas publicaciones se describe entre $70 \%$ y $90 \%$ de éxito quirúrgico, con diferentes criterios de evaluación y plazos de seguimiento. Se sabe que los pacientes con poliposis severa 
o con antecedentes de asma o alergia a la aspirina, presentan mayores tasas de recidiva $^{14,15,17,20,25-28}$.

Debido a esta disparidad en los resultados y criterios en la literatura, el objetivo del presente trabajo fue conocer los resultados de CE en poliposis nasosinusal en el Servicio de Otorrinolaringología del Hospital Barros Luco-Trudeau.

\section{MATERIAL Y MÉTODO}

Se realizó un estudio retrospectivo descriptivo mediante revisión de fichas clínicas de los pacientes sometidos a CE en este servicio. Se incluyeron todas las cirugías de poliposis nasosinusal practicadas entre el año 1999 y noviembre de 2008, con un seguimiento mínimo de 3 meses. A la cantidad inicial de pacientes $(n=278)$, se excluyeron 98 fichas debido a datos incompletos, empleo de otras técnicas quirúrgicas y cirugías practicadas por pólipo antrocoanal.

En estos 180 pacientes se determinó la edad, sexo, motivo de consulta, grado de poliposis, estadificación mediante tomografía computarizada (TC), presencia de tabaquismo, tratamiento pre y posoperatorio, factores asociados (asma, intolerancia a la aspirina; ambos, junto con poliposis nasal, constituyen la 'triada de Sampter'), cirugías previas, tipo y año de cirugía, si la cirugía fue realizada por un médico especialista 0 residente, complicaciones y reoperaciones.

Se registró el grado de poliposis según Lund y $\mathrm{col}^{7}$ al examen físico o mediante examen nasofibroscópico o endoscópico. Se evaluó el compromiso de los senos previo a la cirugía con TC, clasificándose según la estadificación de Kennedy ${ }^{8}$.

La cirugía endoscópica fue encasillada en base al concepto de cirugía progresiva, es decir, según el lugar anatómico hasta el cual se resecó. Es decir, hasta etmoides anterior, hasta etmoides posterior 0 , hasta esfenoides $2,9,10,28$.

Se definió como 'recidiva' a la visualización mediante examen físico, nasofibroscópico 0 endoscópico de lesiones pediculadas en la mucosa nasal después de 6 meses de la cirugía y, se estableció como 'meses libre de enfermedad' a aquel período transcurrido desde la cirugía hasta su último control sin recidiva.
Estudiamos la asociación de recidiva con: presencia de enfermedades, tabaquismo, cirugías previas, grado de poliposis, estadificación según la TC, tipo de cirugía y tratamiento posquirúrgico.

Se realizó el análisis estadístico mediante la estimación de odds ratio (OR) para variables dicotómicas y la prueba de Fisher para variables categóricas con un nivel de confianza de 0,05. Se estimó la tasa de recidiva y los meses libres de recidiva según el método de Kaplan-Meier. Los datos fueron procesados mediante el programa estadístico SPSS 17 para Windows.

\section{RESULTADOS}

Se analizaron 180 CE por poliposis rinosinual entre los años 1999 y 2008. Durante los primeros años su frecuencia fue aumentando progresivamente, para luego mantenerse constante a partir del año 2002, con un promedio de 13 cirugías por año.

Se encontró $63 \%$ de varones y $37 \%$ de mujeres con una diferencia estadísticamente significativa ( $p$ $<0,001$ ). El promedio de edad fue de 44,9 años (DS $\pm 14,3$ años, con una mínima de 5 y una máxima de 95 años).

El motivo de consulta más frecuente, con $79 \%$ de los casos, fue la obstrucción nasal.

La exploración endoscópica mostró mayoritariamente poliposis grado 2, en el $58 \%$; y poliposis masiva, en el $34 \%$ (Figura 1). En la TC se observó un compromiso masivo en el $25 \%$, y un compromiso de uno o más senos, en el $85 \%$ (Figura 2).

El $74 \%$ de los pacientes sometidos a CE no tenían patología concomitante; la más frecuente fue asma, con sólo 17\%. El 18\% de los pacientes refirió el antecedente de tabaquismo. Al evaluar el tratamiento preoperatorio se observó una gran variedad de combinaciones pero, en más de $50 \%$, se usó corticoides inhalados solos 0 asociados (Figura 3). De los pacientes sometidos a CE, en el $58 \%$ se realizó polipectomía, uncinectomía, meatotomía maxilar y etmoidectomía anterior bilateral; en el $15 \%$, una etmoidectomía posterior bilateral; $y$, en el $13 \%$, una esfenoidotomía bilateral (Figura 4). El tratamiento posoperatorio más utilizado -en $31 \%$ de los casos- fue mediante corticoides inhalados y antihistamínicos; en el 38\% no se consignó tratamiento. 


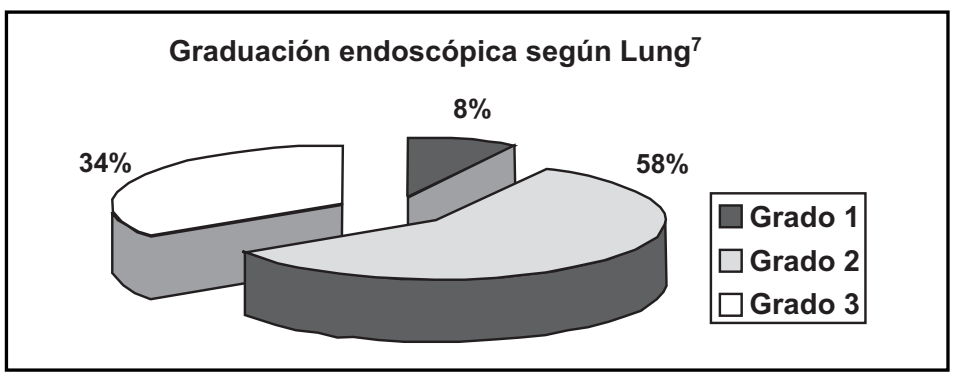

Figura 1. Distribución porcentual de pacientes con poliposis sometidos a CE.

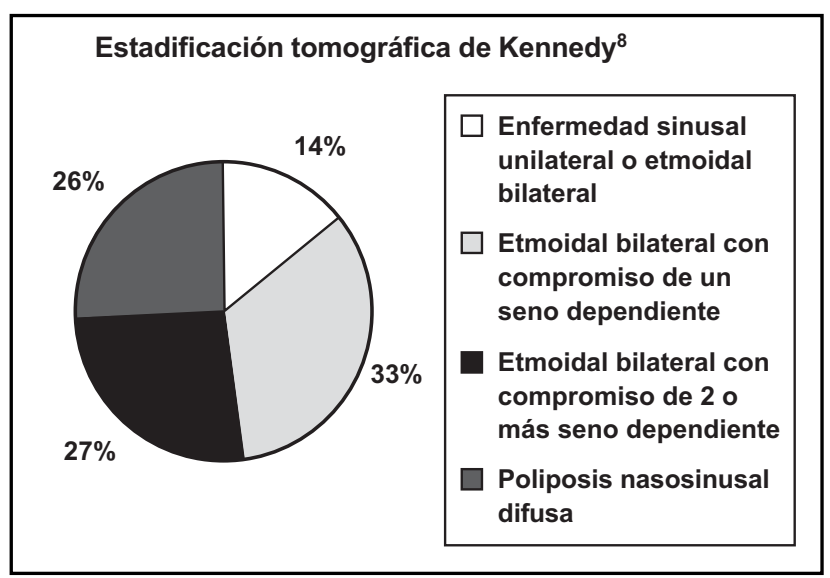

Figura 2. Distribución porcentual de pacientes con poliposis sometidos a CE.

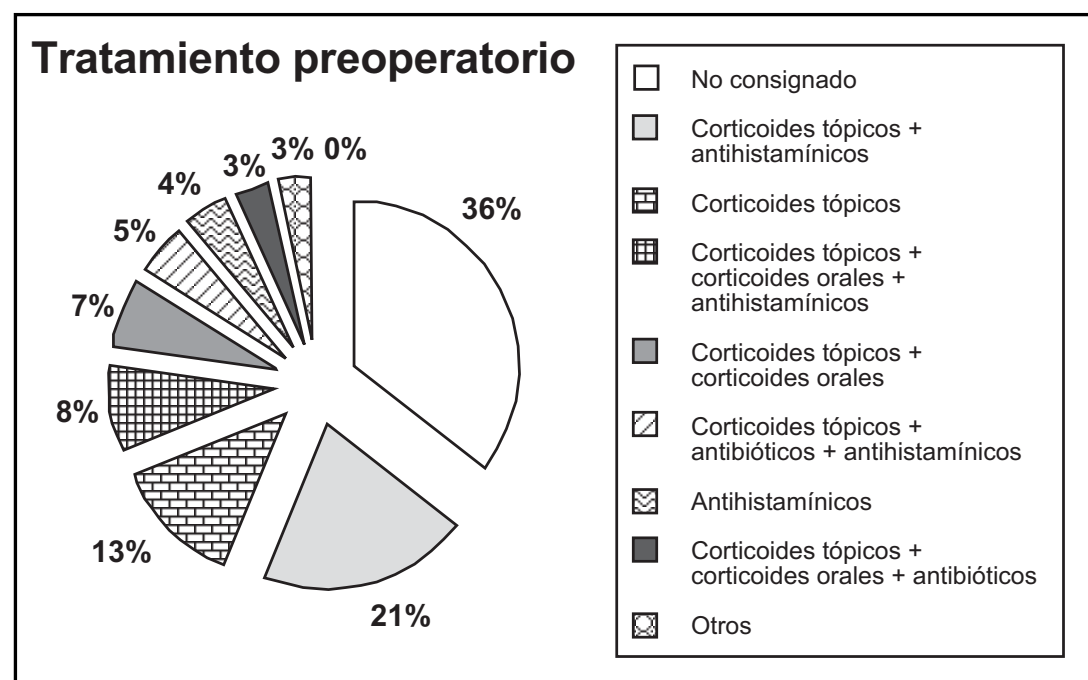

Figura 3. Distribución porcentual de pacientes con poliposis sometidos a CE. 


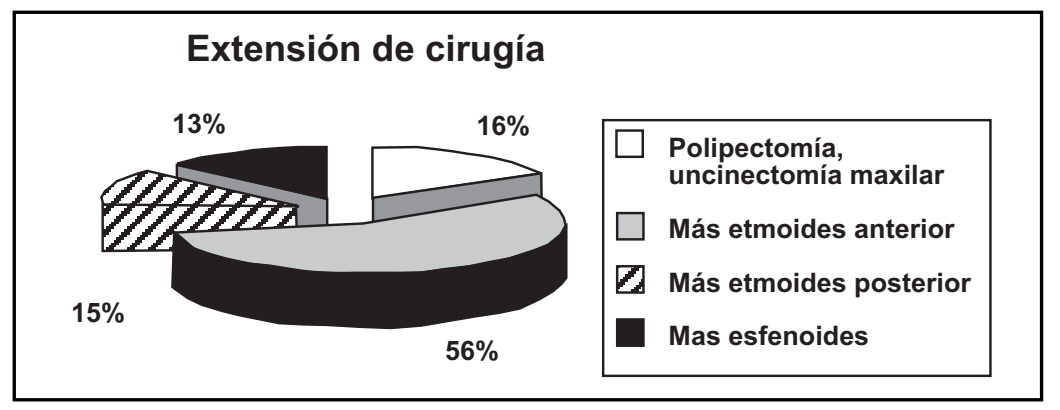

Figura 4. Distribución porcentual de pacientes con poliposis sometidos a CE.

No hubo complicaciones mayores en esta serie estudiada. Las complicaciones menores afectaron a 16 pacientes (9\%). Destacó, como complicación más frecuente, la hemorragia (4\%), siendo necesaria una revisión en pabellón en 4 de estos pacientes. Se presentó una fístula de líquido cerebroespinal (1\%) que fue reparada en el intraoperatorio con buena evolución posoperatoria.

Se observó una pérdida progresiva del seguimiento de los pacientes, siendo únicamente 11 casos quienes completaron los 60 meses de seguimiento posoperatorio (Figura 5).

La recidiva global para los 180 pacientes fue del $24 \%$, la que varió según el tiempo de seguimiento: a los 12 meses, $23 \%$; a los 36 meses, $9 \%$; y, a los 60 meses, $36 \%$ de recidiva (Figura 6).

Se estimó los meses libres de recidiva según el método de Kaplan-Meier, observándose una relación directamente proporcional, es decir, que a mayor tiempo de seguimiento hubo mayor riesgo de recidiva, con un promedio de meses libre de recidiva de 46,7 meses (IC 95\%: 37,5-55,5). Además, según Kaplan-Meier la recidiva al año es de $25 \%$, lo cual es concordante con lo reportado en la literatura (Figura 7).

Se requirió reoperar al $8 \%$ de los pacientes sometidos a CE debido a una recidiva sin respuesta a tratamiento médico.

Se estudió el riesgo de recidiva de poliposis en presencia de tríada de Sampter (OR: 2,2), cirugía previa (OR: 2,1), intolerancia a la aspirina (OR: 1,6), asma (OR: 1,5), tabaco (OR: 1,3), no siendo estadísticamente significativo (Tabla 1).

Al indagar el riesgo de recidiva en relación a grado endoscópico, estadificación según la TC, tipo de cirugía, y tratamiento posoperatorio, no logró significancia estadística al aplicar la prueba de Fisch (Tabla 2).

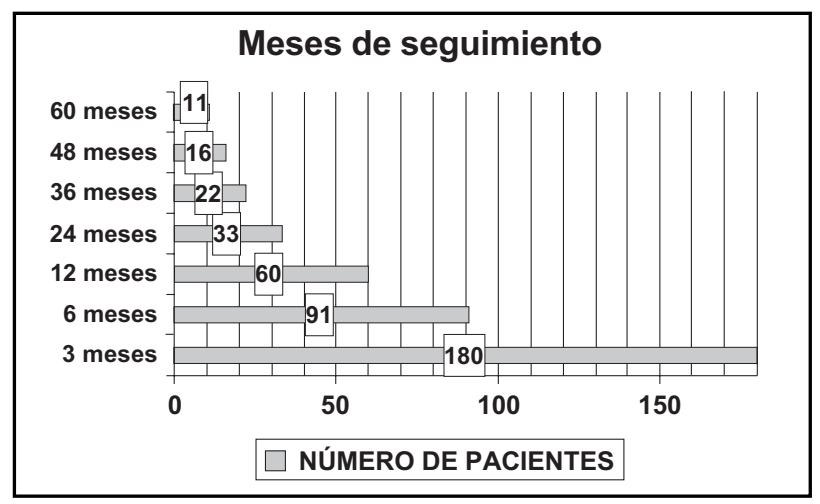

Figura 5. Seguimiento de pacientes con poliposis operados con CE. 


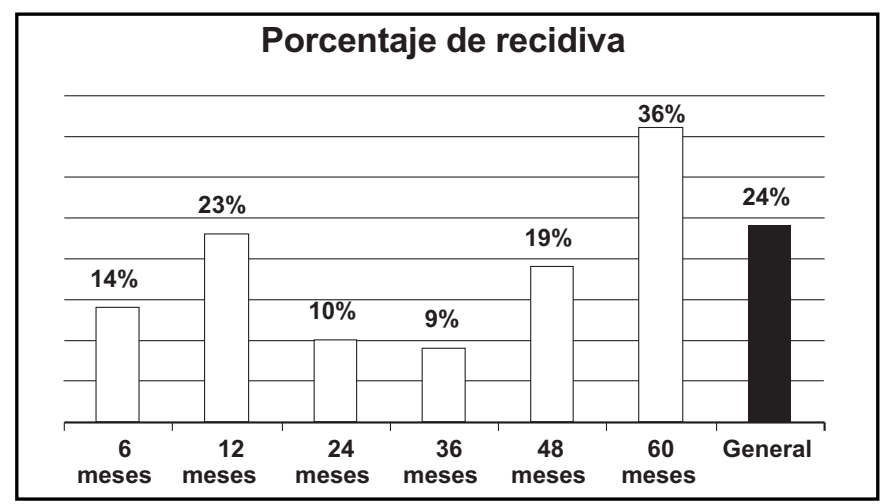

Figura 6. Porcentaje de recidiva según meses de seguimiento.

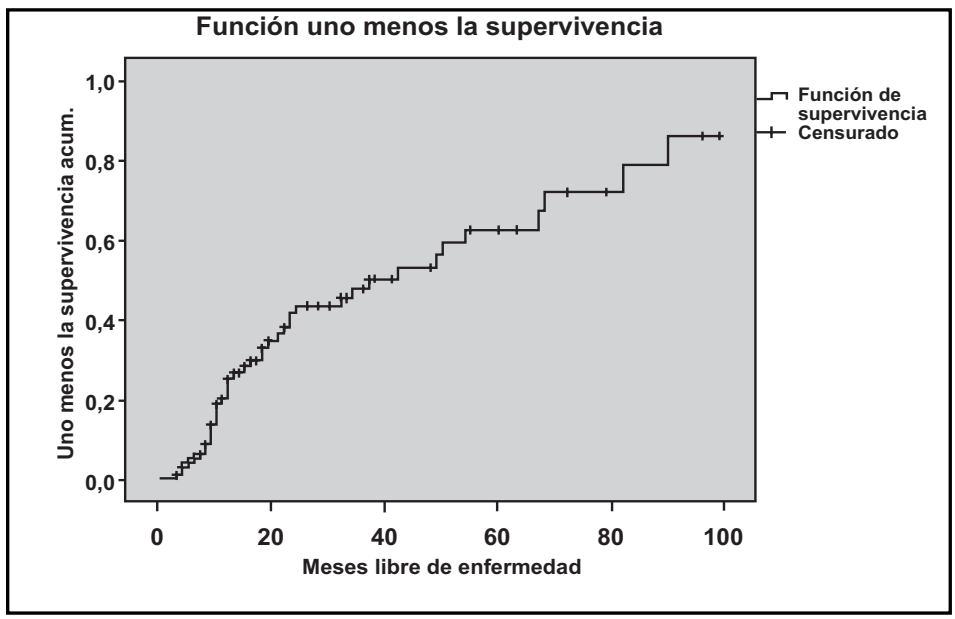

Figura 7. Estimación de los meses libres de recidiva según método de Kaplan-Meier.

Se calculó el riesgo de complicaciones según la ausencia de cirugía previa $(0 R: 0,9)$ y tipo de cirujano (OR: 1,8), tampoco encontrándose significancia estadística (Tabla 3).

\section{DISCUSIÓN}

Se analizaron 180 pacientes operados de poliposis rinosinusal mediante cirugía endoscópica. Las características demográficas, al igual que el predominio del sexo masculino y edad media de la vida de estos pacientes fueron similares a la literatura ${ }^{1-5}$.

El motivo de consulta predominante fue la obstrucción nasal, debido a que más del $80 \%$ de los pacientes tenía grado 2 ó 3 de poliposis, asociado 0 no a otros síntomas rinosinusales ${ }^{1-5}$.

La frecuencia de asma encontrada es similar a lo publicado, si bien existen series con mayor frecuencia de asma cuando se asocia a intolerancia a la aspirina. El porcentaje de ésta resultó ser inferior a lo encontrado en la literatura, ya que por ser un diagnóstico clínico pudo estar subdiagnosticada. Actualmente el patrón de oro (Gold Standard) para el diagnóstico de reacciones respiratorias y cutáneas por la aspirina es el test de provocación oral, el cual puede presentar severas reacciones adversas ${ }^{29}$.

En la literatura se asocia clásicamente la presencia de asma, intolerancia a la aspirina, grado de 
Tabla 1. Estimación de $\mathrm{OR}$ de factores asociados a recidiva

\begin{tabular}{|lccc|}
\hline Factor & Odds ratio & $\mathrm{P}^{*}$ & Intervalo de confianza \\
\hline Tríada de Sampter & 2,2 & 0,21 (NS) & $0,5-8$ \\
Cirugía previa & 2,1 & 0,05 (NS) & $1-4,5$ \\
Intolerancia a la aspirina & 1,6 & 0,29 (NS) & $0,5-5$ \\
Asma & 1,5 & 0,23 (NS) & $0,7-3$ \\
Tabaco & 1,3 & 0,37 (NS) & $0,5-2,9$ \\
\hline
\end{tabular}

${ }^{*}<0,05, \mathrm{NS}=$ no significativo

Tabla 2. Asociación de factores estudiados con recidiva

\begin{tabular}{|lcc|}
\hline Factor & Valor de Fisher & Valor de $\mathrm{p}^{*}$ \\
\hline Grado endoscópico de poliposis & 0,2 & $\mathrm{p}=0,96$ (NS) \\
Estadificación según tomografía computarizada. & 0,6 & $\mathrm{p}=0,92$ (NS) \\
Tipo de cirugía & 2,1 & $\mathrm{p}=0,71$ (NS) \\
Tratamiento posoperatorio & 4,2 & $\mathrm{p}=0,38$ (NS) \\
\hline
\end{tabular}

${ }^{*}<0,05, \mathrm{NS}=$ no significativo

Tabla 3. Estimación de OR de factores asociados a complicación

\begin{tabular}{|lccc|}
\hline Factor & Odds ratio & $\mathrm{p}^{*}$ & Intervalo de confianza \\
\hline Sin cirugía previa & 0,9 & 0,59 (NS) & $0,2-3,3$ \\
Residente & 1,8 & 0,22 (NS) & $0,5-6$ \\
\hline
\end{tabular}

${ }^{*}<0,05, \mathrm{NS}=$ no significativo

poliposis y estadificación de la TC con un mayor riesgo de recidiva poscirugía ${ }^{2,25}$. En el presente estudio, si bien existe mayor riesgo, éste no alcanza a ser estadísticamente significativo.

Al analizar los pacientes previamente operados no se observa que se asocie a un mayor riesgo de recidiva, al igual que en otras publicaciones ${ }^{14}$.

Al evaluar el tabaquismo como factor de riesgo en la recidiva de poliposis, éste no fue estadísticamente significativo, a pesar que su hábito sugeriría un peor resultado. No se dispone de trabajos controlados que así lo demuestren ${ }^{24}$.
El tipo de cirugía, entendiéndose la CE como una cirugía progresiva que reinstaura la ventilación y transporte mucociliar a través de los orificios naturales, permitiendo que se recupere la mucosa sinusal patológica, no presenta mayor riesgo de recidiva al intervenir sólo los senos comprometidos. Llama la atención que no hubo necesidad de ampliar el receso del frontal en nuestros pacientes $2,9-11$.

En esta revisión la terapia poscirugía no se asocia de manera estadísticamente significativa a mayores tasas de recidiva, a pesar que en la 
literatura el uso de terapia médica posquirúrgica, especialmente corticoides, puede tener un impacto significativo en la evolución posoperatoria ${ }^{13,18,20}$.

Es probable que la pérdida de seguimiento y la baja prevalencia de enfermedades asociadas en nuestro estudio expliquen la falta de asociación estadísticamente significativa con factores clásicamente descritos.

Se encontró una variedad de esquemas de tratamientos pre y posoperatorios, pero la mayoría se basó en corticoides tópicos solos 0 asociados. Estos, por sus múltiples efectos en las células y mediadores inflamatorios de la mucosa nasal, han demostrado eficacia clínica en el control de la poliposis nasal16,17,21,22. En la mayoría de los pacientes con rinosinusitis crónica con pólipos, un tratamiento médico adecuado es tan eficaz como una intervención quirúrgica. La cirugía sinusal se debe reservar para los pacientes que no respondan satisfactoriamente al tratamiento médico ${ }^{2-4,13,16}$.

La recidiva se definió en forma objetiva ya que es conocido que los pacientes se sienten mejor de lo que se observa en la mucosa nasal ${ }^{13}$. La recidiva general fue de $24 \%$. Cuando se analizó según períodos, a los 12 meses de seguimiento se observó $23 \%$ de recidiva; a los 36 meses, $9 \%$; y a los 60 meses, $36 \%$ de recidiva, siendo similar a lo encontrado en la literatura de acuerdo a los tiempos de seguimiento ${ }^{26-28}$.

Según el método de Kaplan-Meier, al aumentar el tiempo de seguimiento, la probabilidad de recidiva se incrementa en forma lineal, lo cual es concordante con el hecho de que la poliposis es una enfermedad de la mucosa, donde interactúan múltiples factores. La cirugía es una estrategia más en el control de la enferme$\operatorname{dad}^{13,20,21}$.

El hecho que el promedio de recidiva se sitúe alrededor de los 4 años nos hace reflexionar que el seguimiento de nuestros pacientes debe ser estrecho, por lo menos anual, ya que sólo en contadas ocasiones algunos pacientes no presentarán recidiva a largo plazo ${ }^{13,21}$.

De los pacientes que recidivaron sólo 8\% debió ser reoperado, cifra similar a lo reportado en otras series, ya que el resto respondió bien al tratamiento médico ${ }^{2,13,14}$.
El porcentaje total de complicaciones fue similar a la literatura, y en la presente serie no se presentaron complicaciones mayores. La fístula de líquido céfalo-raquídeo fue considerada menor porque no tuvo complicaciones y se reparó en el mismo acto quirúrgico, al igual que los sangrados. De éstos, ninguno requirió transfusión ni hubo lesión de algún vaso arterial importante ${ }^{14,28}$.

Las cirugías previas o el nivel de experiencia del cirujano no se asociaron a mayores complicaciones a pesar que en la literatura sí se relacionan ${ }^{4-5,14,28}$. Lo más probable es que, en el caso del nivel de experiencia del cirujano, los residentes operan junto a un médico especialista quien los guía y que, obviamente, posee más experiencia.

\section{CONCLUSIONES}

En nuestro servicio la CE es un procedimiento seguro y eficaz al igual que en otros centros. Representa, en la actualidad, la mejor opción quirúrgica para el manejo de la poliposis.

Al evaluar las recidivas no se encontraron factores de mal pronóstico que fueran estadísticamente significativos.

El perfil de los pacientes que consultan nuestro servicio es de un nivel socioeconómico bajo, el que no permite una buena adherencia al tratamiento médico posoperatorio; a pesar de ello, se obtuvieron tasas de recidiva similares a la literatura.

Nuestro número de cirugías anuales permite un adecuado entrenamiento quirúrgico.

Se requiere optimizar el seguimiento de nuestros pacientes y la disponibilidad de tratamiento médico posoperatorio, para un mejor control de esta enfermedad.

\section{BIBLIOGRAFÍA}

1. Toledano A, Herráiz C, Navas C. et al. Estudio epidemiológico en pacientes con poliposis nasal. Acta Otorrinolaringol Esp 2008; 59(9): 438-43. 
2. Fokkens W, Lund V, Mullol J; European position PAPER ON RHINOSINUSITIS AND NASAL POLYP GROUP. European position paper on rhinosinusitis and nasal polyps 2007. Rhinology 2007; 45 (suppl 20): $1-136$.

3. Fokkens W, Lund V, Bachert C, et al. eAACl Position paper on rhinosinusitis and nasal polyp executive summary. Allergy 2005; 60: 583-601.

4. Bachert C, Hörmann K, Mösges R, et al. An update on the diagnosis and treatment of sinusitis and nasal polyposis. Allergy 2003; 58: 176-91.

5. Meltzer e, Hamilos d, Hadley J et al. Rhinosinusitis: Establishing definitions for clinical research and patient care. Otolaryngol Head Neck Surg 2004; 131(suppl) (6): S1S61.

6. Fonseca X. Poliposis nasal ¿una enfermedad alérgica? Rev Otorrinolaringol Cir Cabeza y Cuello 1989; 49: 55-62.

7. LUND VJ, MackaY I. Staging in rhinosinusitis. Rhinology 1993; 31: 183-4.

8. Lund VJ, Kennedy DW. Staging for rhinosinusitis. Otolaryngol Head Neck Surg 1997; 117: S35-S40.

9. Emhart C, Emhart M. Cirugía endoscópica sinusal funcional revisión de 315 casos. Rev Otorrinolaringol Cir Cabeza Cuello 1994; 54: 9-17.

10. Kennedy DW. Functional endoscopic sinus surgery: Technique. Arch Otolaryngol 1985; 111: 643-9.

11. Stammberger $H$. Surgical treatment of nasal polyps: past, present, and future. Allergy 1999; 54(suppl 53): 7-11.

12. Bhattacharyya N. Influence of polyps on outcomes after endoscopic sinus surgery. Laryngoscope 2007; 117: 1834-8.

13. Alobid I, Benítez P, Bernal-Sprekelsen M. Nasal polyposis and its impact on quality of life: comparison between the effects of medical and surgical treatments. Allergy 2005: 60: 452-8.

14. Llorente Jl, Martínez-Farreras A, Rodrigo JP, ET AL. Poliposis nasal: Resultados a los
5 años tras cirugía endoscópica nasal. Acta Otorrinolaringol Esp 2002; 53: 102-9.

15. Guerreo J, Molina B, Echeverria L, Arribas I, Rivera T. Cirugía endoscópica nasosinusal: Estudio de 110 pacientes con rinosinusitis crónica con pólipos. Acta Otorrinolaringol Esp 2007; 58(6): 252-6.

16. Benitez P, Alobid I, De Haro J. A short course of oral prednisone followed by intranasal budesonide is an effective treatment of severe nasal polyps. Laryngoscope 2006; 116: $770-5$.

17. Dufour X, Bedier A, Ferrie J. Diffuse nasal polyposis and endonasal endoscopic surgery: Long-term results, a 65-case study. Laryngoscope 2004; 114: 1982-7.

18. Zer Toros S, BölüKbasi S, Naiboflu B. Comparative outcomes of endoscopic sinus surgery in patients with chronic sinusitis and nasal polyps. Eur Arch Otorhinolaryngol 2007; 264: 1003-8.

19. Wright E, Agrawal S. Impact of perioperative systemic steroids on surgical outcomes in patients with chronic rhinosinusitis with polyposis: evaluation with the novel perioperative Sinus Endoscopy (POSE) Scoring System. Laryngoscope 2007; 117(suppl. 115): 1-28.

20. Batra P, Kern R, Tripathi A. Outcome analysis of endoscopic sinus surgery in patients with nasal polyps and asthma. Laryngoscope 2003; 113: 1703-6.

21. BECKER S. Surgical management of polyps in the treatment of nasal airway obstruction. Otolaryngol Clin North Am 2009; 42: 37785.

22. Bıкhazı N. Contemporary management of nasal polyps. Otolaryngol Clin North Am 2004; 37: 327-37.

23. Newton JR, Ah-See KW. A review of nasal polyposis. Therapeutics and clinical risk management. Ther Clin Risk Manag 2008; 4(2): 507-12.

24. Houser S, Keen K. The role of allergy and smoking in chronic rhinosinusitis and polyposis. Laryngoscope 2008; 118: 1521-7. 
25. Wynn R, HaR-El G. Recurrence rates after endoscopic sinus surgery for massive sinus polyposis. Laryngoscope 2004; 114: 811-3.

26. Garrel R, Gardiner Q, Khudjadze M et al. Endoscopic surgical treatment of sinonasal polyposis-medium term outcomes (mean follow-up of 5 years). Rhinology 2003; 41 (2): 91-6.

27. Poetker DM, Mendolia-Loffredo S, Smith TL. Outcomes of endoscopic sinus surgery for chronic rhinosinusitis associated with sinonasal polyposis. Am J Rhinol 2007; 21 (1): 84-8.

28. Dalziel K, Stein K, Round A, Garside R, Royle P. Endoscopic sinus surgery for the excision of nasal polyps: A systematic review of safety and effectiveness. Am J Rhinol 2006; 20 (5): 506-19.

29. Gajardo P, Fonseca X. Intolerancia a la aspirina en pacientes con poliposis nasal $y$ asma bronquial. Rev Otorrinolaringol Cir Cabeza Cuello 2009; 69: 157-68. 